International Journal on Applied Bioengineering, Vol. 5 No. 2 July 2011

\title{
STRUCTURE BASED DRUG DESIGN OF LUNG CANCER EGFR PROTEIN INHIBITORS
}

\author{
Ravikumar M., Jeyanthi Rebecca L., Xavier Suresh M., Ananthi Rachel L. \\ Department of Bioinformatics Sathyabama University, Chennai-600 119 \\ Tamil Nadu India
}

\section{ABSTRACT}

The epidermal growth factor receptor (EGFR) is under investigation as a therapeutic target for cancers. Lung cancer cell lines are variably dependent on autocrine stimulation of EGFR since it has a role in signal transduction .We therefore examined the effects of a selective EGFR tyrosine kinase inhibitor zd1839 (iressa drug).Using chemskecth I have designed 2 new lead molecules "iressa I, iressa II" on the basis of iressa drug, and compared both compounds by docking it on EGFR protein by using vega zz software. On the basis of energy score I found that iressa I will be more effective inhibitor than iressa II .Further on comparing the ADME properties of iressa I and iressa by using ADME tool, I found that this new drug having fluorine molecule in place of chlorine molecule will act as more efficient inhibitor than original iressa drug to block the activity of EGFR protein and stop signal transduction. This new drug which $I$ named as iressa $I$ is an effective inhibitor and can be test for its effectiveness in clinical trials to cure lung cancer.

Keywords: Pneumonetomy, chemotherapy, radiotherapy

\section{INTRODUCTION}

The activities of epidermal growth factor receptor (EGFR) have been identified as key drivers in the process of cell proliferation, metastasis, angiogenesis, and dedifferentiation. It has been shown that the EGFR-mediated drive is increased in a wide variety of solid tumors including lung cancer, prostate cancer, breast cancer, gastric cancer, and tumors of the head and neck. Heightened activity at the EGFR is caused by an increase in the concentration of ligand around the cell, an increase in receptor numbers or receptor mutation can lead to an increase in the drive for the cell to replicate. It has been postulated that agents designed to block EGFR will inhibit signal transduction, resulting in multiple antitumor mechanisms, as well as enhancing chemotherapy and radiotherapy antitumor effects.

\section{LUNG CANCER-CAUSES AND TREATMENT}

Lung cancer is usually caused by smoking or exposure to second-hand smoke. Researcher estimates that more than $90 \%$ of lung cancer in men and at least $70 \%$ in women are caused by cigarette smoking.

Normally, there is a thin layer of mucus and thousands of tiny hairs(cilia) lining the inside of our breathing tubes within our lungs. The mucus and cilia act as a natural cleaning system for our lungs.If we breath in dirty or polluted air, the little bits of dirt, the cilia move together like a wave to push the dirt-filled mucus out of your lungs. Then we cough, spit up, or swallow the mucus, and the dirt is out or your lungs.

Smoke from cigarettes, cigars and pipes is made up of over 4,000 dangerous chemicals; many of these chemicals are carcinogens .smoking damages our lungs by delivering these chemicals inside our body , and causes damage to lungs and effects its natural cleaning and repair systems. Thus dirt and pollution get stuck into lungs, and stay there. As dirt and smoke chemicals bulid up in lungs, increases the higer change of getting lung cancer. The more you smoke, or exposed to cigarette smoke,the greater your risk of getting lung cancer. Depending upon what kind of lung cancer you had, and what stage cancer is it can be treated with:-

(i) Surgery: Pneumonetomy, Lobectomy, Segmenectomy, Laser surgery

(ii) Radiation therapy: It uses high energy radiation to kill cancer cells.

(iii) Chemotherapy: It means taking anti-cancer drugs.

(iv) Targeted therapy: This is a word for drugs that attack pathways specifics for cancer cells 


\section{ROLE OF EPIDERMAL GROWTH FACTOR RECEPTOR IN LUNG CANCER}

The EGFR is a transmembrane receptor with an extracellular ligand-binding domain, a helical transmembrane domain, and an intracellular tyrosine kinase domain. Activation of EGFR by epidermal growth factor (EGF) and other ligands (amphiregulin, TGF-) which bind to its extracellular domain is the first step in a series of complex signaling pathways which take the message to proliferate from the cell membrane to the genetic material deep within the cell nucleus.

Epidermal growth factor (EGF) is a small mitogenic protein that is thought to be involved in mechanisms such as normal cell growth, oncogenesis, and wound healing.

EGF is a small 53 amino acid residue long protein that contains three disulfide bridges. As described in the preceding sections, epidermal growth factor receptor (EGFR) has been shown to play a critical role in regulating tumor cell growth, repair and survival, angiogenesis, invasion, and metastasis, and is expressed in a significant percentage of human tumors.

\section{EGFR-TARGETED ANTI-CANCER THERAPIES}

The biology of epidermal growth factor receptor (EGFR) suggests its potential as a target for anti-cancer therapy. Biologic therapy targeted at aberrant pathways that are unique to, or dysregulated in, tumor cells is expected to be more selective and less toxic than conventional cytotoxic therapy.

(i) Anti-EGFR antibodies: the antibody binds to the cell surface EGFR resulting in internalization and degradation of the receptor-antibody complex

(ii) Tyrosine kinase (TK) inhibitors inhibitors bind to EGFR TK intracellularly, inhibiting kinase activity and blocking signal transduction

(iii) Ligand-toxin and immunotoxin conjugates ligand-toxin conjugates comprise an EGFR ligand conjugated with toxin, and internalization of the complex results in the inhibition of protein synthesis and cell death.

(iv) Antisense oligonucleotides: EGFR or transforming growth factor (TGF)- $\alpha$ antisense oligonucleotides are targeted to DNA or RNA, and ultimately prevent protein synthesis and promote cell death.

(v) EGFR/ligand-directed vaccines - stimulate the production of antibodies that bind EGFR/ligand and enhance the systemic immune response.

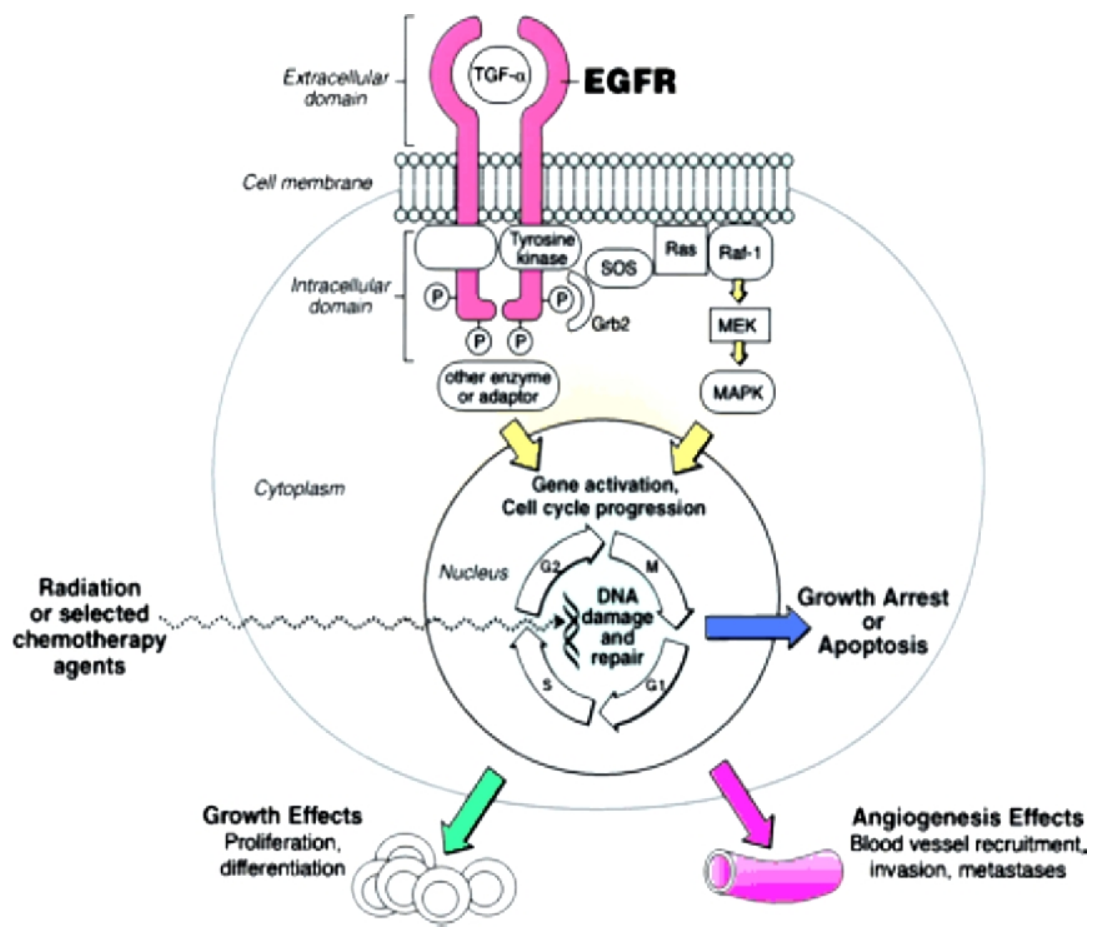

Fig. 2 EGFR signaling pathway. 
Currently available options for the treatment of many solid tumors are limited primarily to standard chemotherapies and radiation. In a significant portion of the patient population, these agents are not effective in eradicating disease, especially in late stages. This may be due in part to the ability of EGFR to repair damage in tumor cells that are not killed by these agents.

\section{STRUCTURE BASED DRUG DESIGNING (SBDD)}

Structure-based drug design, the three-dimensional structure of a drug target interacting with small molecules is used to guide drug discovery. "Structure-based drug design represents the idea that we can see exactly how our molecule interacts with its target protein". SBDD helps to get quality molecules, molecules that have better pharmacological chemical properties. You have a much greater awareness of how these compounds bind to the pocket, so you have a much greater awareness of what parts of the molecule you can modify on the basis original drug chemical structure. The active site of the protein was a space to be filled with a molecule that complemented it in terms of shape, charge, and other binding components.

\section{MATERIALS \& METHODS}

\section{(i) Receptor:-Epidermal Growth Factor Receptor} Protein

\section{PDB ID: - 1 MOX}

Title: - Crystal Structure of Human Epidermal Growth Factor Receptor (residues 1-501) in complex with TGF-alpha

\section{(ii) Standard ligand (Iressa) for EGFR}

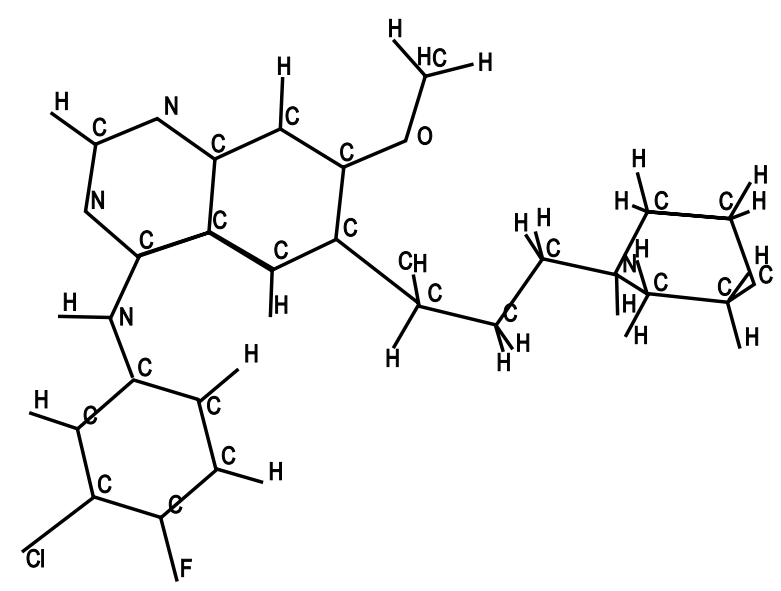

Fig. 3 Structure of Iressa

\section{(iii) Chemsketch software}

$\mathrm{ACD} /$ ChemSketch is the powerful all-purpose chemical drawing and graphics package from ACD/Labs developed to help chemists quickly and easily draw molecules, reactions, and schematic diagrams, calculate chemical properties, and design professional reports and presentations. It includes structure mode for drawing chemical structures and calculating their properties. Draw mode or text and graphics processing. Structure mode, we can perform the following actions draw chemical structures using the buttons located on the Structure toolbar, Atoms toolbar and References toolbar. By using this software I have designed two inhibitors on the basis of chemical formula of Iressa drug and named it as Iressa I \& Iressa II.

\section{(a) Structure of Iressa I}

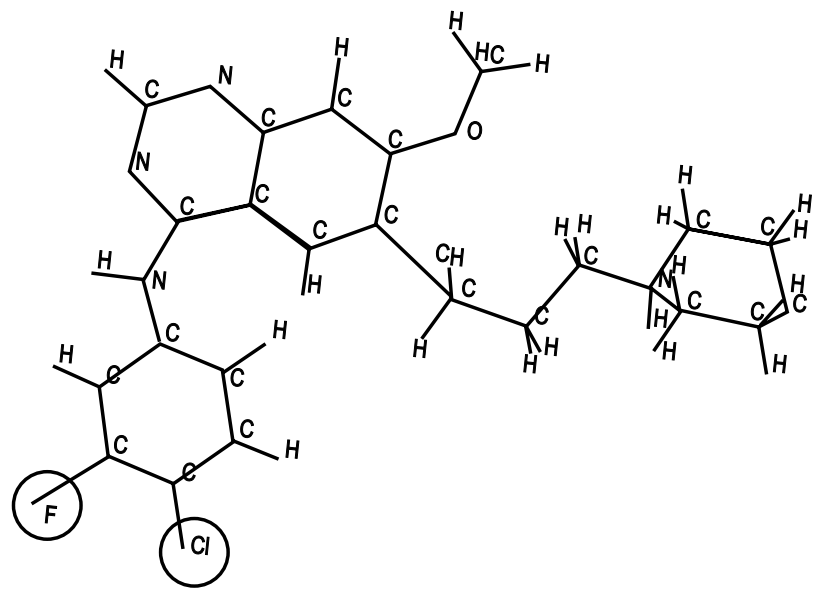

Fig. 4 Structure of Iressa I

\section{(b) Structure of Iressa II}

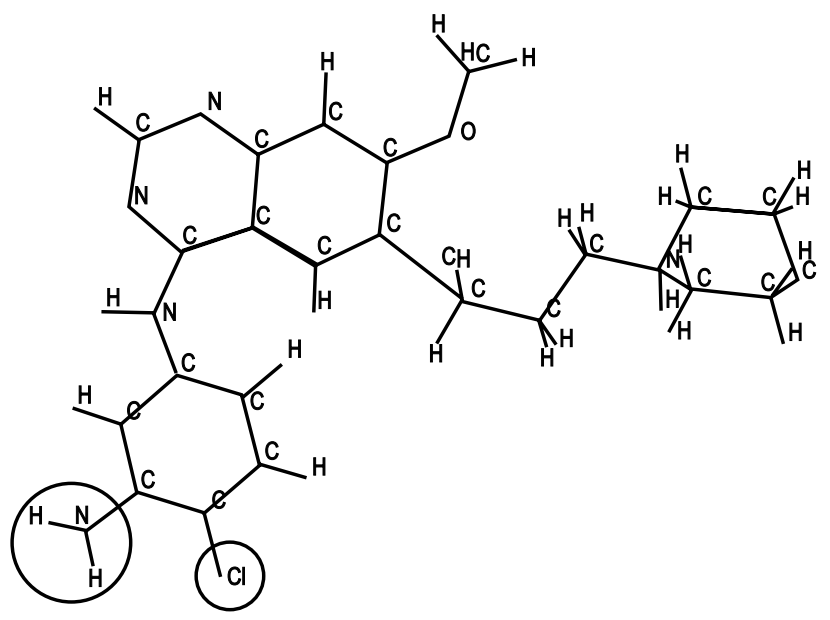

Fig. 5 Structure of Iressa II 


\section{(iv) VegazZ 2.0.5 Software for Docking}

It is molecular modeling software and includes several new features and enhancements making your research jobs very easy.

(a) EscherNG V1.0.2:- ESCHER Next Generation (NG) is a new docking procedure named as "evaluation of surface complimentarity, hydrogen bonding and electrostatic interaction in molecular recognition". Escher consists of three modules that work in series to perform docking procedure. ESCHER is a rigid docking program, written in $C$ language. The three modules and their work to perform docking are as following:

(i) SHAPES module: evaluates the geometric complementarity and produces a set of rough solutions for the docking problem.

(ii) BUMPS module: identifies molecular collisions within those solutions

(iii) CHARGES module: this module evaluates their electrostatic complementarity

(v) ADMETox: ADME-Tox (poor absorption, distribution, metabolism, elimination (ADME) or toxicity) filtering for small compounds. Based on a set of elementary rules called as Lipinski rule of five which states that absorption or permeation of drug is impaired when: $-\log P>5$, Molecular weight $>500$, number of hydrogen donar groups $>5$, number of hydrogen acceptor groups $>10$

\section{METHODOLOGY}

(i) Docking: Docking of the iressa, iressa I and iressa II inhibitor to EGFR protein

(ii) Solution file: Solutions are generated by docking inhibitors to EGFR protein, once docking is completed we can generate pdb file of solutions and can interpret the result by using energy graph.

(iii) Observe minimum conformation molecule: Docking score includes; Score, rms, bumps values, charges etc.

(iv) Output lowest energy conformation of the iressa I \& II inhibitor

(v) Comparative Analysis: After generating the solution file of all inhibitors compare the score and minimum energy conformation molecule of all two inhibitors and find out the best inhibitor having minimum energy conformation.

(vi) ADME property: compound having minimum energy configuration is checked for its ADME property by using ADMETox tool

(vii) Compared with original iressa molecule to test its effectiveness.

\section{RESULTS}

Docking result of Iressa I and Iressa 2

\section{(i) Interaction energy graph of Iressa I}

100 models are generated after Docking iressa inhibitor to target protein and the best conformation is

$\begin{array}{ll}\text { Best Model: } & 1 \\ \text { Best Score: } & 266 \\ \text { Rmsd: } & 70.0 \\ \text { Bumps: } & 0\end{array}$

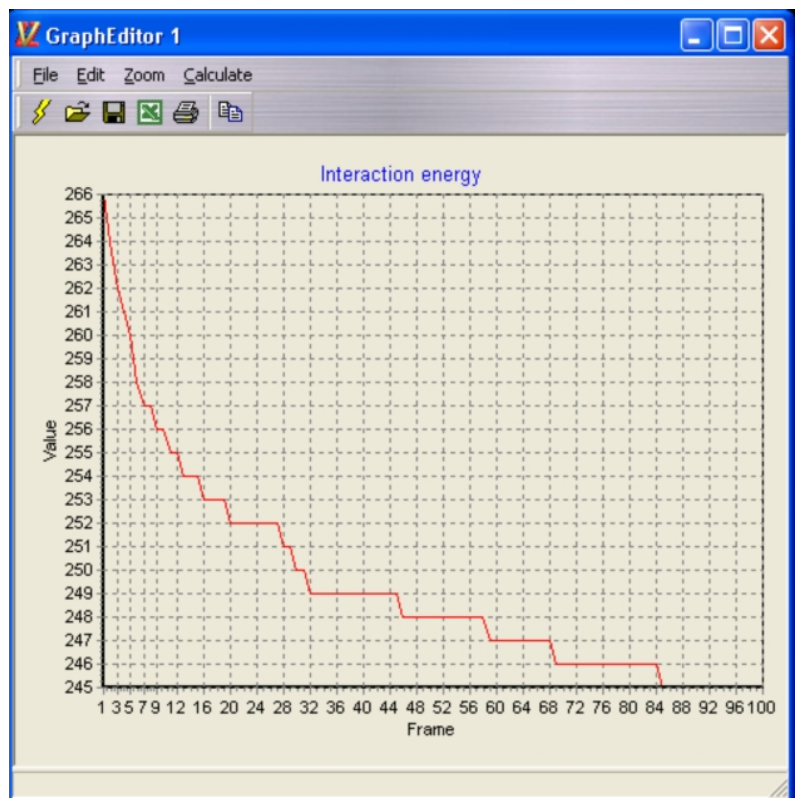

Fig. 6 Interaction energy graph of Iressa I

\section{(ii) Interaction energy graph of Iressa II}

100 models are generated after Docking iressa inhibitor to target protein and the best conformation is:

$\begin{array}{ll}\text { Best Model: } & 1 \\ \text { Best Score: } & 280 \\ \text { Rmsd: } & 70 \\ \text { Bumps: } & 0\end{array}$




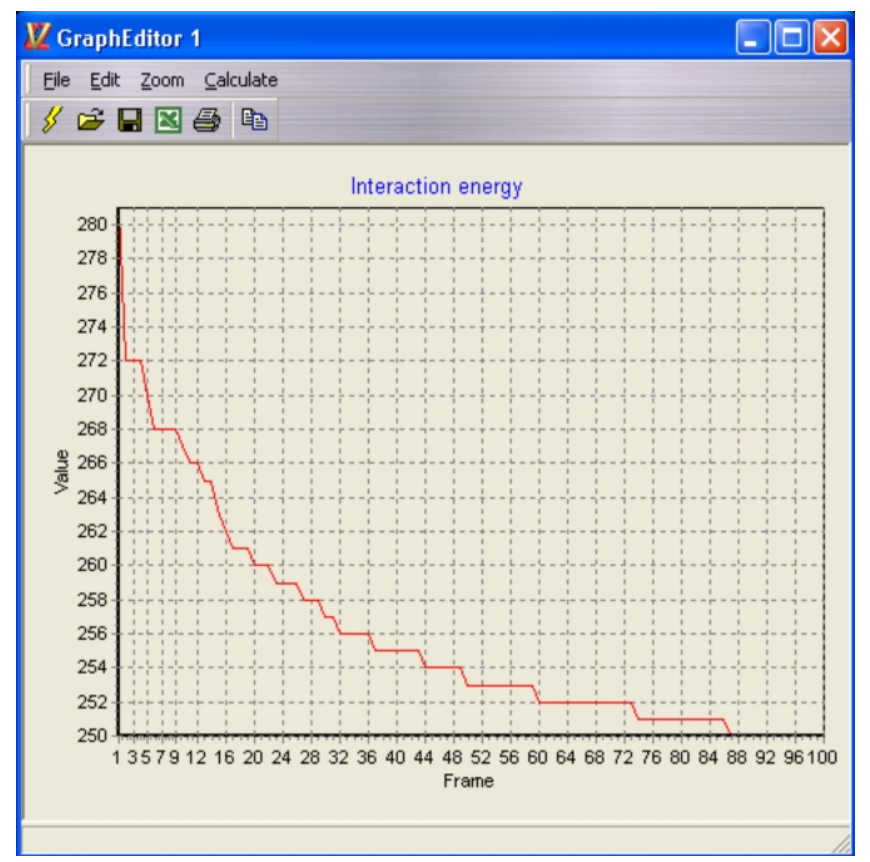

Fig. 7 Interaction energy graph of Iressa II

On comparing the interaction energy of Iressa I \& Iressa II. Iressa II is the best inhibitor since highest the score lower is the energy so according to energy graph and the energy ranges it is concluded that iressa II is the best inhibitor having the highest energy score of 280.0

\section{(iii) Interaction of Iressa II to EGFR receptor}

The iressa II compound interacts with 535 residues first residue is leucine and last is valine, and 4101 atoms. (iv) ADME property of Iressa II: Input smiley of iressa II to ADMETox c1([c]2[c](ncn1) $\operatorname{cc}(\mathrm{c}(\mathrm{OCCCN} 3 \mathrm{CCOCC} 3) \mathrm{c2}) \mathrm{OC}) \mathrm{Nc} 4 \mathrm{cc}(\mathrm{c}(\mathrm{Cl}) \mathrm{cc} 4) \mathrm{F}$

(a) Output

MW : 446.7, Drs : 1, Ars : 7, FB : 8, RB : 23, \#R : 4, RL : 6, C : 22, nC : 9, C/nC : 0.409091, \#Chrg : 0, Chrg : 0, LogP : 3.180000, PSA : 68.740000

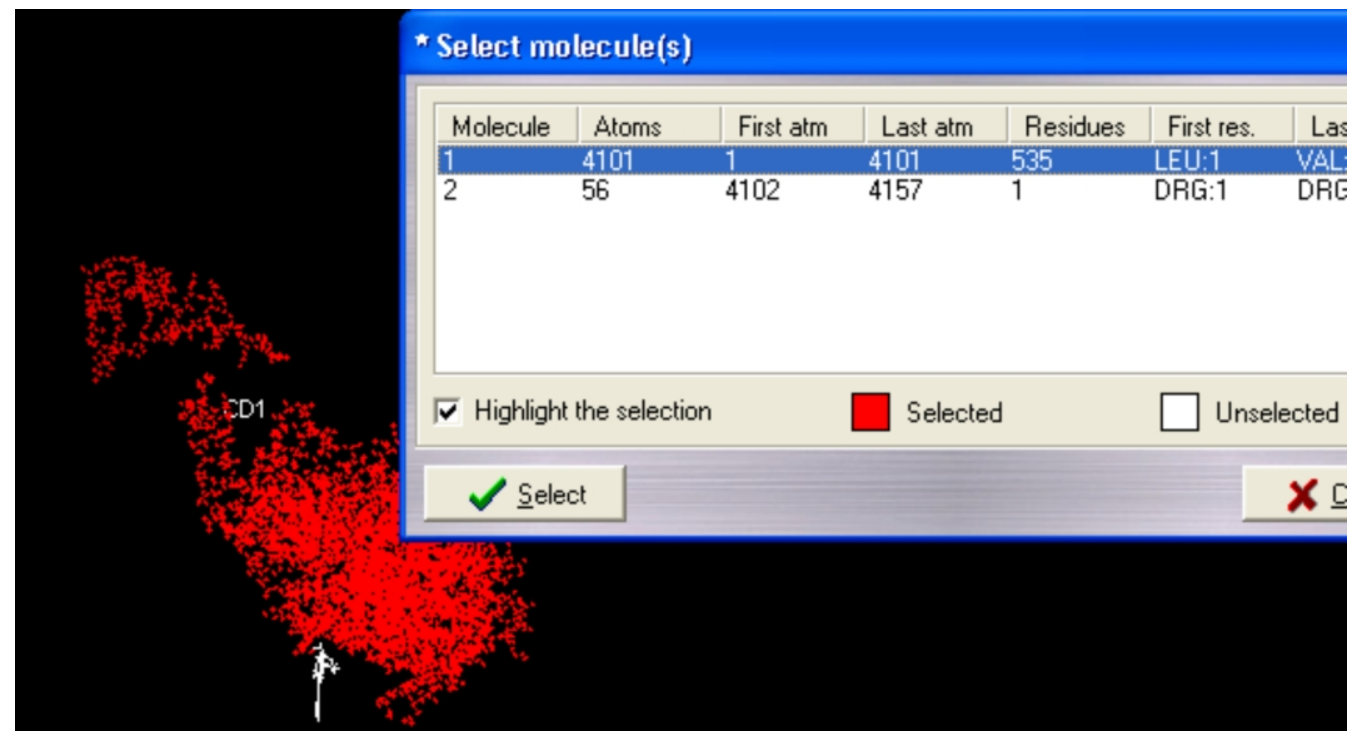

Fig. 8 Docking result of Iressa I 
MW : Molecular weight, Drs : Donors, Ars : Acceptors, FB : flexible bonds, RB : Rigid Bonds, \#R : Ring Number, RL : Ring Length, $\mathbf{C}$ : carbons, $\mathbf{n C}$ : non carbons, $\mathrm{C} / \mathrm{nC}$ : ratio non carbons/carbons, \#Chrg : number of charges, Chrg: Total Charge, LogP : $\log P$ (octanol / water), PSA: Polar surface area.

(b) Remarks: The above result of ADMETox shows that Iressa compound follows the Lipinski rule of five that have been already described in text. So by determining the molecular weight, number of hydrogen donors, acceptors, LogP we can determine the absorption and permeation phenomena of compound .

\section{CONCLUSION}

The biology of epidermal growth factor receptor (EGFR) suggests its potential as a target for anti-cancer therapy. Tyrosine kinase (TK) inhibitors bind to EGFR TK intracellularly, inhibiting kinase activity and blocking signal transduction This new drug "Iressa I" is an effective inhibitor than original Iressa molecule and can be test for its effectiveness in clinical trials to cure lung cancer.

\section{REFERENCES}

[1] Abdel-Meguid, S., Zhao, B., Murthy, K.H.M., Winborne, E., Choi, J.-K., Desjarlais, R.L., Minnich, M.D., Culp, J.S., Debouck, C., Tomaszek, T.A.,Inhibition of human immunode ${ }^{2}$ ciency virus-1 protease by a C2-symmetric phosphinate. Biochemistry, 32,
[2] Bacon, D.J. \& Moult, J. (1992). Docking by least-squares @iting of molecular surface patterns. J. Mol. Biol. 225, 849858.

[3] Bernstein, F.C., Koetzle, T.F., Williams, G.J.B., Meyer, E.T.J., Brice, M.D., Rodgers, J.R., Kennard, O.,

[4] Carlson HA, McCammon JA: Accommodating protein flexibility in computational drug design. Mol Pharmacol 2000, 57:213-218. 27.

[5] Claussen $H$, Buning $C$, and Rarey $M$, Lengauer $T$ : FlexE: efficient molecular docking considering protein structure variations. J Mol Biol 2001, 308:377-395.

[6] H. Kubinyi, Combinatorial and computational approaches in structure-based drug design, Curr. Opin. Drug Discov. Develop. 1 (1998) 16-27.

[7] J. Bajorath, Selected concepts and investigations in compound classification, molecular descriptor analysis, and virtual screening, J.Chem. Inf. Comput. Sci. 41 (2001) 233-245.

[8] L. Xue, J. Godden, F.L. Stahura, J. Bajorath, A dual fingerprint based metric for the design of combinatorial libraries and analogs, J. Mol. Mod. 7 (2001) 125-131.

[9] P. Labute, Binary QSAR: a new method for the determination of quantitative structure activity relationships, Pac. Symp. Biocomput. 7 (1999) 444-455.

[10] Y. Jin, T.M. Penning, Steroid 5-_-reductases and 3_-hydroxysteroid dehydrogenases: key enzymes in androgen metabolism, Best Pract. Res. Clin. Endocrinol. Metabol. 15 (2001) 79-94. 